\title{
INTERFACE SLIP CAUSED BY A SURFACE LOAD MOVING AT CONSTANT SPEED
}

\author{
Maria Comninou \\ Department of Civil Engineering, University of Michigan. Ann Arbor, MI 48109, U.S.A. \\ J. R. B ARBER \\ Department of Mechanical Engineering and Applied Mechanics, University of Michigan, Ann \\ Arbor, MI 48109, U.S.A. \\ and \\ JoHN DUNDURS \\ Department of Civil Engineering, Northwestern University, Evanston, IL 60201, U.S.A.
}

(Received 24 February 1982; in revised form 14 May 1982)

\begin{abstract}
Summary - Slip at the interface between a layer and a substrate caused by a slowly moving tangential force on the surface of the layer is considered. The solution shows a significant departure from the stationary load case. In particular, a permanent tangential displacement is left behind the moving load, symmetry is lost, and the shear tractions have continuous derivatives at the trailing edge of the transition zone.
\end{abstract}

\section{INTRODUCTION}

The problem of frictional slip and separation between a layer and a substrate has been considered in recent publications [1-3]. In particular the cases of an elastic layer pressed uniformly on a substrate of similar material properties and subjected to a concentrated normal or tangential force at a fixed point on the surface of the layer have been studied. For a normal force two symmetric slip zones develop at the interface and expand as the magnitude of the force increases [1]. If the normal force is tensile, the two slip zones eventually coalesce and separation occurs at the center of the newly formed single slip zone[2]. For a tangential force, a single slip zone develops first behind the force at the interface [3]. As the magnitude of the force is increased a second slip zone appears ahead of the force at the interface and separation occurs inside the first slip zone. Which of these events happens first depends on the magnitude of the coefficient of friction. For low friction the second slip zone develops before separation occurs. For high friction separation appears inside the first slip zone before the second slip zone is formed.

A related problem of practical interest is the effect of a load moving over the surface of the layer. If the load moves sufficiently slowly for inertia effects to be neglected, it might be thought that the solution would be identical to that for a stationary load [1-3] since the equations to be satisfied are identical, but this is not so because the inequality requiring the frictional traction to be in the direction opposing slip has different implications for the two problems. Suppose we focus attention on a point fixed in space at the interface. The tractions will increase as the load approaches and decrease after it passes overhead and recedes. Thus, we have in effect a loading and unloading problem and if slip occurs during loading, the stress field during unloading will depend upon the loading history. Indeed, we would anticipate that the moving load would leave behind a permanent tangential displacement of the layer with respect to the half-plane.

No such displacement is possible with the stationary loading problem and in [1-3] this fact furnishes an equation which is needed to determine the unknown extent of the slip region. With the moving load, this equation is lost, but in its place we find that the inequalities impose a more stringent condition on the asymptotic behavior of the stress and displacement fields at the trailing edge of the slip zone.

Whatever symmetry existed in the static problem, is now lost. Thus, the 
parameters specifying the extent and location of the slip and separation zones increase in number and the iteration procedure used for their determination is, in consequence, more complicated.

To simplify matters we consider here the steady-state case of a tangential load moving sufficiently slowly for the inertia effects to be neglected, and we limit the magnitude of the force so that only one slip zone occurs. The formulation and analysis is the same as in the static problem[3]. For this reason many details are omitted, but in each step relevant expressions are quoted for completeness.

\section{FORMULATION}

Consider an elastic layer with shear modulus $\mu$ and Poisson's ratio $\nu$ pressed against a half plane of similar elastic properties by a uniform pressure $p_{0}$. The thickness of the layer is denoted by $h$. A tangential force of magnitude $Q$ moves steadily on the surface of the layer with constant speed $c$. We consider a stationary frame of reference $(\hat{x}, \hat{y})$ and a frame of reference $(x, y)$ moving with velocity $c$ along the interface as shown in Fig. 1. Then

$$
x=\hat{x}-c t, \quad y=\hat{y} .
$$

For very low values of the loading parameter $Q / p_{0} h$ the interface does not slip anywhere and the quasistatic solution coincides with the static Flamant solution. The tractions at the interface are then

$$
\begin{gathered}
\sigma_{x y}(x, 0)=\frac{2 Q}{\pi} \frac{h x^{2}}{\left(h^{2}+x^{2}\right)^{2}} \\
\sigma_{y y}(x, 0)=-p_{0}-\frac{2 Q}{\pi} \frac{h^{2} x}{\left(x^{2}+h^{2}\right)^{2}}
\end{gathered}
$$

and the following inequalities are satisfied

$$
\begin{gathered}
\sigma_{y y}(x, 0)<0 \\
\left|\sigma_{x y}(x, 0)\right|<f\left|\sigma_{y y}(x, 0)\right|
\end{gathered}
$$

where $f$ is the coefficient of friction. As the magnitude of $Q / p_{0} h$ is increased slip starts at a point the Fosition of which depends on the coefficient of friction (see Fig. 2 of [3]). We assume that for a given value of $Q / p_{0} h$ the slip zone occupies the interval $(-a,-b)$ where $a$ and $b$ are unknowns. The slip zone is modeled by a distribution of glide dislocations with density $B_{x}(x)$ and the total shear $S(x)$ and normal $N(x)$ tractions at the interface are [3]

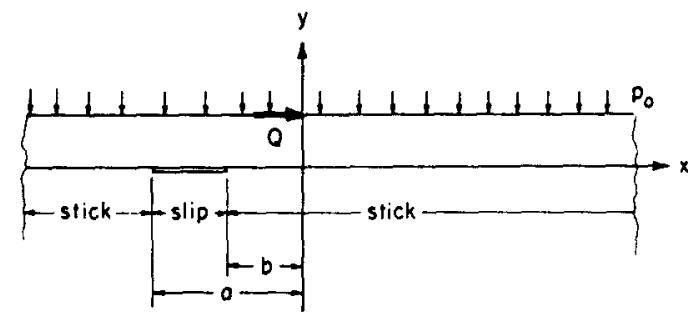

FIG. I. Geometry of the problem in the moving frame of reference.

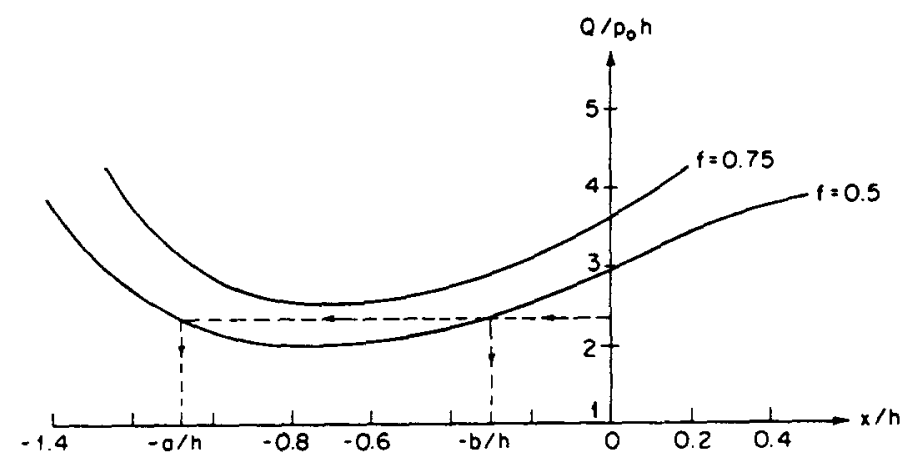

FIG. 2. Extent and location of slip zone for given load. 


$$
\begin{gathered}
S(x)=\frac{2 Q}{\pi} \frac{h x^{2}}{\left(h^{2}+x^{2}\right)^{2}}+\frac{2 \mu}{\pi(\kappa+1)} \int_{-a}^{-b} \frac{B_{x}(\xi) \mathrm{d} \xi}{x-\xi}+\frac{2 \mu}{\pi(\kappa+1)} \int_{-a}^{-b} B_{x}(\xi) K_{1}(x, \xi) \mathrm{d} \xi \\
N(x)=p_{0}-\frac{2 Q}{\pi} \frac{h^{2} x}{\left(h^{2}+x^{2}\right)^{2}}+\frac{2 \mu}{\pi(\kappa+1)} \int_{-a}^{-b} B_{x}(\xi) K_{2}(x, \xi) \mathrm{d} \xi
\end{gathered}
$$

where

$$
\begin{gathered}
K_{1}(x, \xi)=-\frac{x-\xi}{4 h^{2}+(x-\xi)^{2}}+\frac{12 h^{2}(x-\xi)}{\left[4 h^{2}+(x-\xi)^{-2}\right]^{2}}-\frac{64 h^{4}(x-\xi)}{\left[4 h^{2}+(x-\xi)^{2}\right]^{3}} \\
K_{2}(x, \xi)=-\frac{24 h^{3}}{\left[4 h^{2}+(x-\xi)^{2}\right]^{2}}+\frac{128 h^{5}}{\left[4 h^{2}+(x-\xi)^{2}\right]^{3}}
\end{gathered}
$$

and $\kappa=3-4 \nu$ for plane strain. The dislocation density $B_{r}(x)$ is related to the tangential displacement discontinuity or relative shift $h(x)$ by

$$
B_{x}(x)=-\frac{\mathrm{d} h(x)}{\mathrm{d} x}
$$

The boundary conditions to be satisfied at the interface are

$$
\begin{gathered}
|S(x)|=f|N(x)| \quad-a<x<-b \\
N(x)<0 \quad-\infty<x<\infty \\
|S(x)|<f|N(x)| \quad x>-b, \quad x<-a \\
\operatorname{sgn} S(x)=\operatorname{sgn} V(x)
\end{gathered}
$$

where $V(x)$ is the slip velocity defined as

$$
V(x)=\frac{\mathrm{d} h(x)}{\mathrm{d} t}=c B_{x}(x)
$$

Since the shear traction $S(x)$ is expected to be positive in the slip zone, we may tentatively write eqn (11) as

$$
S(x)=-f N(x) \quad-a<x<-b
$$

where (12) was used. Conditions of continuity and boundary conditions on the surface of the layer are automatically satisfied by this formulation. A net relative shift $D$ is anticipated between the layer and the substrate given by

$$
D=\int_{-a}^{-b} B_{x}(\xi) \mathrm{d} \xi
$$

It is worth pointing out the differences from the static solution. In the static solution $D$ must vanish so that (17) is an auxiliary condition required by the solution. In addition, eqn (14) is replaced by

$$
\operatorname{sgn} S(x)=\operatorname{sgn} h(x) .
$$

Since the static solution satisfied (18) with a bell-shaped $h(x)$, it cannot satisfy (14) as seen from (15). This suggests that the condition $D=0$, lost in the moving load problem, is to be replaced by a condition obtained from the inequalities. At this point it is enlightening to study the asymptotics of the transition between slip and stick. For the static case the relevant results can be found in [4] $\dagger$ and can be used directly provided that $x, y$ are interpreted as moving coordinates and $V(x)$ is given by (15). We find that for a slip zone moving between regions of stick equations (13) and (14) give a stronger result compared to the static case, but for the trailing end of the slip zone only. The result is that the shear tractions at the trailing transition point must have continuous derivatives. In contrast, the asymptotics allow for discontinuous derivatives (infinite slope on the side of the stick zone) at the leading end of the slip zone, and cause the familiar "hooks" on the shear tractions which are present at both ends of a slip zone surrounded by stick zones in static situations.

\section{RESULTS}

Substituting (6) and (7) into (16) we obtain a singular integral equation of the Cauchy type. For its discretization we use the method of Erdogan and Gupta [5]. The detailed equations are given in [3]. To simplify the numerical iterations required for the determination of the parameters $a$ and $b$ which appear nonlinearly in the equation, we interchange the roles of $b$ and $Q / p_{0} h$. Thus we consider $b$ as given and treat $Q / p_{0} h$, which appears linearly in the discretized system, as unknown. We then have to iterate only for the

†Note the misprints in [4]. Equations (3.18) and (3.32) should read $h(x)=-M C r^{1-\lambda} \sin \lambda \pi$ and $h(x)=$ $M \mathrm{Mr}^{2-\lambda_{0}} \sin \lambda_{0} \pi$ respectively. 


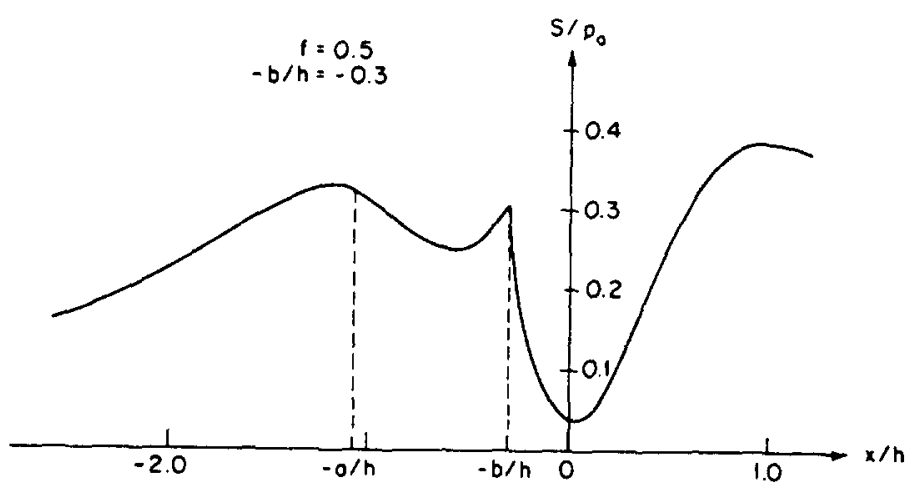

FIG. 3. Shear tractions for $f=0.5,-b / h=-0.3$.

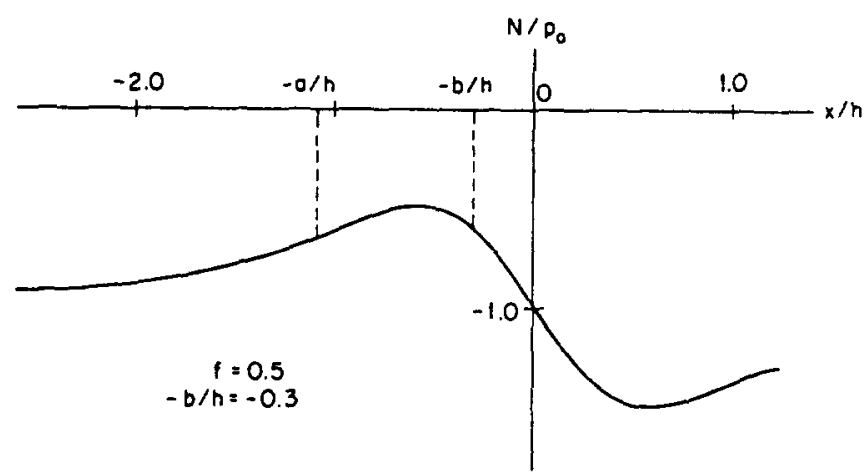

FIG. 4. Normal tractions for $f=0.5,-b / h=-0.3$.

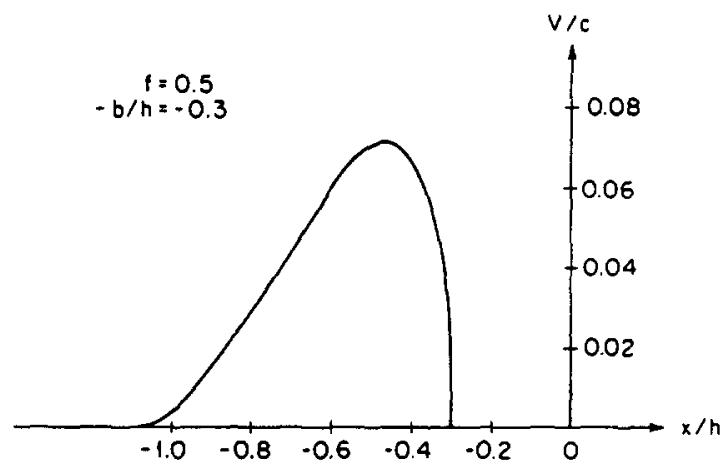

FIG. 5. Slip velocity for $f=0.5,-b / h=-0.3$.

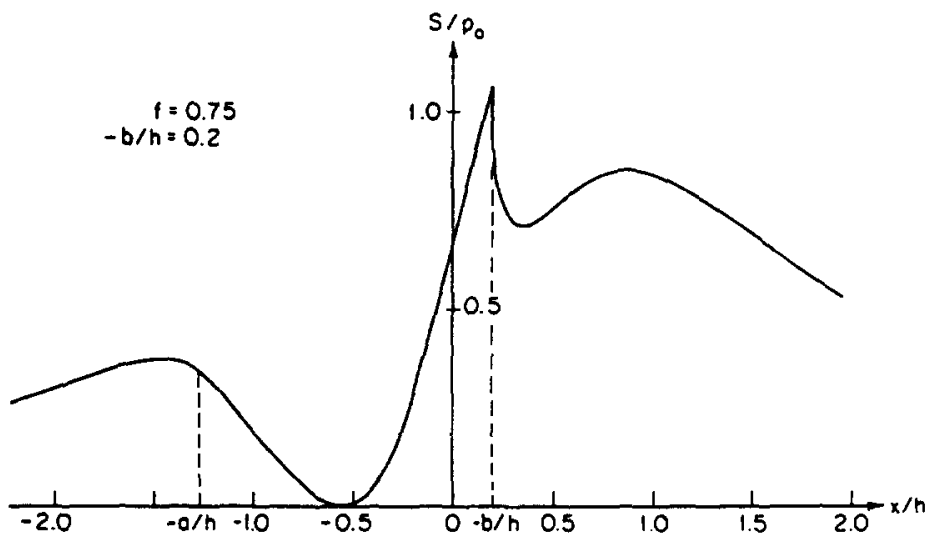

FIG. 6. Shear tractions for $f=0.75,-b / h=0.2$ (incipient separation). 


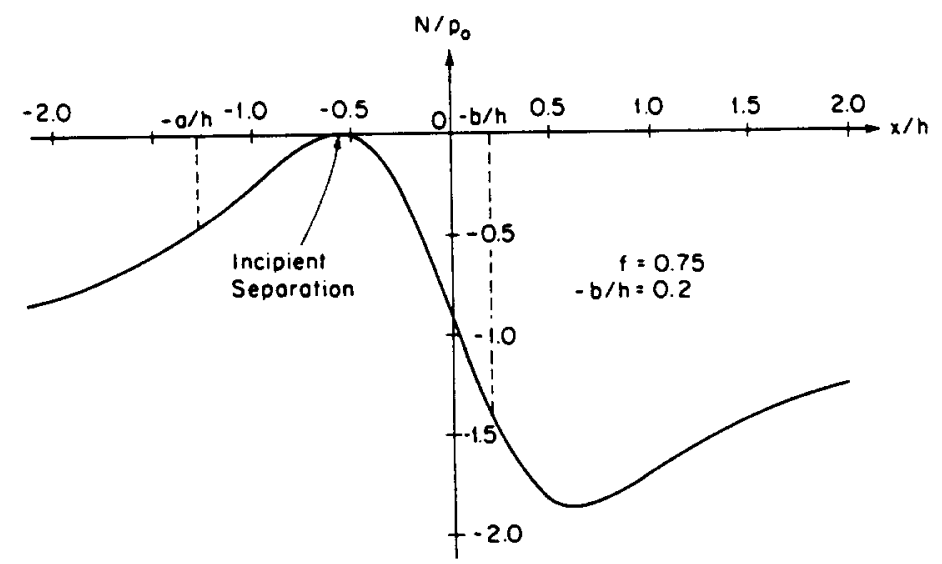

FIG. 7. Normal tractions for $f=0.75,-b / h=0.2$ (incipient separation).

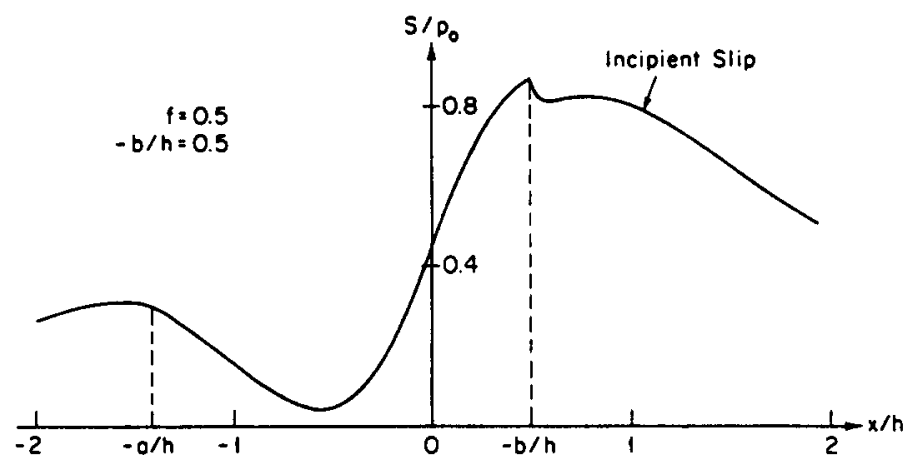

FIG. 8. Shear tractions for $f=0.5,-b / h=0.5$ (incipient slip).

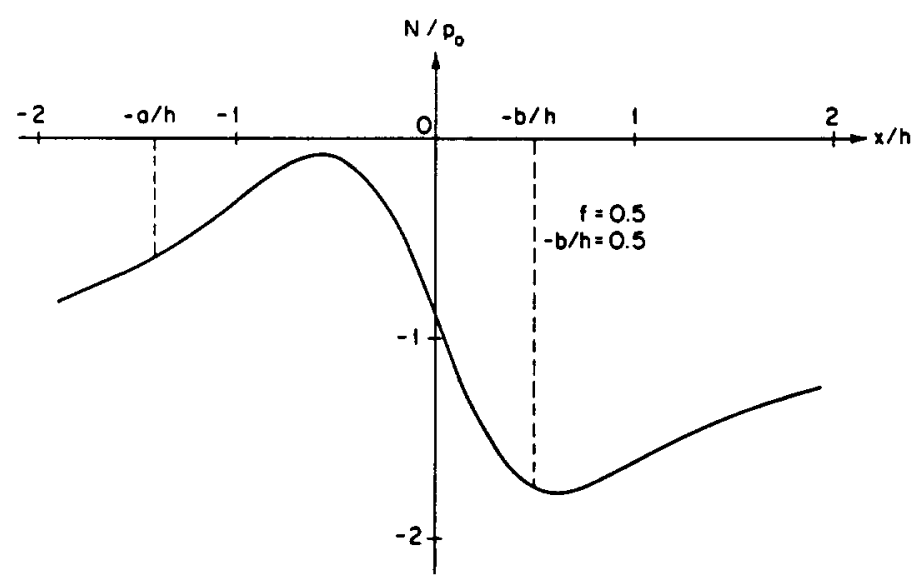

FIG. 9. Normal tractions for $f=0.5,-b / h=0.5$ (incipient slip).

parameter $a$. If the value guessed for $a$ is too big in magnitude we find that (14) is violated. If the value guessed for $a$ is too small in magnitude we find that (13) is violated. In this way we are able to determine a unique value of $a$ for which all the conditions of the problem are satisfied.

Representative results are shown in Figs. 2-10. The extent and location of the slip zone for given $Q / p_{0} h$ and for two values of the coefficient of friction can be determined from Fig. 2. The curve corresponding to $f=0.75$ ends where separation is about to occur in the slip zone (that is, the next increment in $-b$ would cause tensile normal tractions in the slip zone) and the curve corresponding to $f=0.5$ stops where a second slip zone is about to form ahead of the force $Q$ at the interface. In both cases the first slip zone starts at the location and for the load predicted by the Flamant solution. Fig. 3 shows the shear tractions for $f=0.5$ and $b / h=-0.3$. Note the continuous transition at the trailing end of the slip zone and the "hook" at the leading end. Fig. 4 shows the normal tractions and Fig. 5 the slip velocity for the same case. Fig. 6 and Fig. 7 show the shear and normal tractions for the case of incipient separation $(f=0.75,-b / h=0.2)$. Figures 8 and 9 


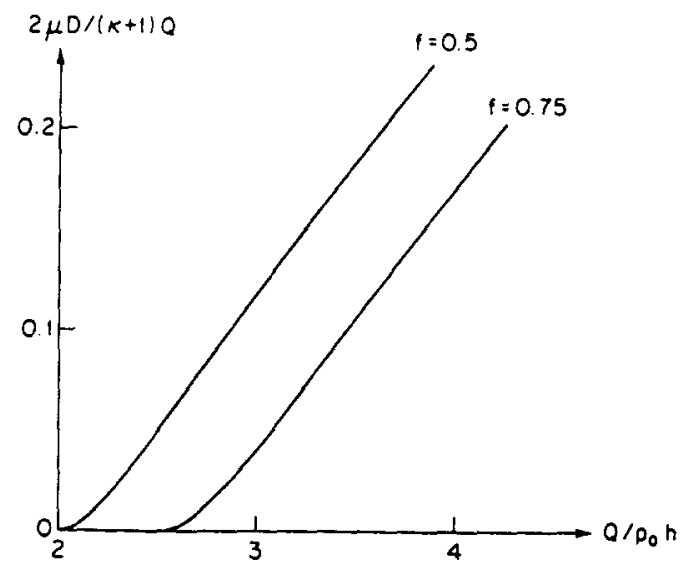

FIG. 10. Dimensionless total shift vs $Q / p_{0} h$.

show the shear and normal tractions for the case of incipient slip ahead of $Q(f=0.5,-b / h=0.5)$. In this case the second slip zone develops before separation occurs in the first. Fig. 10 shows the dependence of the dimensionless total shift $2 \mu D /(\kappa+1) Q$ on the loading parameter $Q / p_{0} h$ for $f=0.5$ and $f=0.75$. The shift vanishes at $Q / p_{0} h$ corresponding to the Flamant solution (stick everywhere).

Acknowledgement-Support by the National Science Foundation under the grant CME8019281 is gratefully acknowledged.

\section{REFERENCES}

1. M. Comninou, D. SChmUeSER and J. Dundurs, Frictional slip between a layer and a substrate caused by a normal load. Int. J. Engng Sci. 18, 131-137 (1980).

2. D. SChmueser, M. Comninou and J. Dundurs, Separation and slip between a layer and a substrate caused by tensile load. Int. J. Engng Sci. 18, 1149-1155 (1980).

3. D. SChmueser, M. Comninou and J. Dundurs, Frictional slip between layers and substrate. J. Engng. Mech. Div., ASCE 107, 1103-1118 (1981).

4. J. Dundurs and M. Comninou, Some consequences of the inequality conditions in contact and crack problems. J. Elasticity 9, 71-82 (1979).

5. F. ERDOGAN and G. GUPTA, On the numerical solution of singular integral equations. Quart. Appl. Math. 30, 525-534 (1972). 